\title{
LA MÉMOIRE MUSICALE ET L'ÉCOLE DE TLEMCEN
}

\author{
Mustapha Guenaou \\ Centre de recherche en Anthropologie Sociale et Culturelle \\ CRASC - Orán
}

\begin{abstract}
Resumen
Este trabajo estudia algunos aspectos relativos al traslado de la memoria colectiva estética y artística de los moriscos a Tremecén, Argelia; antigua capital del Magreb central. Las aportaciones de Maurice Halbwachs sirven de marco teórico para el estudio de las memorias socio-históricas y sociolingüísticas de esta población. En particular, esta contribución se centra en un elemento esencial de la cultura de Tremecén: la música andalusí.
\end{abstract}

Palabras clave: Moriscos, Tremecén, memoria cultural, música andalusí.

\begin{abstract}
This work studies some aspects related to the transfer of the collective aesthetic and artistic memory of the Moriscos to Tlemcen, Algeria; former capital of the central Maghreb. The contributions of Maurice Halbwachs serve as a theoretical framework for the study of the socio-historical and sociolinguistic memories of this population. In particular, this contribution focuses on an essential element of the culture of Tlemcen: andalusian classical music.
\end{abstract}

Keywords: Moriscos, Tlemcen, cultural memory, andalusian classical music.

\section{Résumé}

Ce travail étudie certains aspects liés au transfert de la mémoire esthétique et artistique collective des Morisques à Tlemcen, Algérie ; ancienne capitale du Maghreb central. Les contributions de Maurice Halbwachs servent de cadre théorique pour l'étude des mémoires socio-historiques et sociolinguistiques de cette population. En particulier, cette contribution se concentre sur un élément essentiel de la culture de Tlemcen : la musique arabo-andalouse.

Mots clés: Morisques, Tlemcen, mémoire culturelle, musique arabo-andalouse.

"Pour comprendre l'exil, il faut le vivre."

Cette note est une tentative de la compréhension de l'exil que nombreux sont les intellectuels et autres qui l'ont connu. Elle a pour source la parti- 
cipation à une rencontre scientifique, organisée par un centre de recherche français, ayant pour axe les recherches ibériques ${ }^{1}$.

Sans aucune nostalgie, notre vision est de tenter de mieux comprendre ce que les anciens musulmans d'Espagne ont vécu, avant leur exil vers les pays du Maghreb. Il s'agit, également, de connaître, selon les us et coutumes ${ }^{2}$, l'héritage socio culturel et artistique que nous comptons exposer ${ }^{3}$.

A partir de la mémoire et de l'histoire, nous pouvons avancer, sur un plan intellectuel et socioculturel et surtout rappeler l'existentiel de cette population qui aurait, grâce aux efforts et des échanges, fourni ce que conserve encore la mémoire collective de Tlemcen, ancienne capitale du Maghreb central.

Dans le cadre de notre projet de recherche, dont les résultats ont été agréé par le conseil scientifique du CRAsc-Oran (Centre de Recherche en Anthropologie Sociale et Culturelle), nous avons apporté quelques éclaircissements sur la réalité historique des anciens Andalous, installés au Maghreb. Il ne faut pas oublier ce que cet exil a pu apporter à la population de cette contrée qui se reconnait dans son riche passé d'échange entre l'Europe et l'Afrique du Nord. Les possibilités de création et d'innovation sont, réellement, favorables à ce transfert de la mémoire collective andalouse vers l'Algérie, et plus particulièrement à Tlemcen, ancienne capitale du Maghreb central.

Nous préférons, dans cette contribution, insister, uniquement, sur un élément essentiel de la médina de Tlemcen : la maison traditionnelle que nous lui attribuons l'appellation de maison médinale. Depuis leur arrivée à Tlemcen, la maison médinale connut, principalement, des améliorations dans l'art, la structure et la distribution de ses pièces. La démarche, que nous avons adoptée, est basée sur les principes de la théorie de Maurice Halbwachs. Elle nous conduit à nous intéresser, essentiellement, à la mémoire collective d'une médina ou d'une ville précoloniale, connue pour son riche passé.

1 Ce séminaire est une rencontre scientifique et une initiative du Centre de Recherches Ibériques et Latino-américaines de l'Université de Perpignan.

2 Cf. Rene Maunier, Coutumes algériennes, París, Domat-Montchrestien, 1935 ; et E. Daumas, Mours et coutumes de l'Algérie, París, Sindbad, 1988.

3 Cf. Shynar (Pessah), Islam Maghrébin contemporain, Essai de bibliographie sélective et annotée sur le Maroc, Algérie, Tunisie, Libye, (1830-1978), Paris, CNrs, 1983. 
La question de départ nous permit d'élaborer la problématique suivante : Quels sont les avantages recensés, en rapport avec les repères socio anthropologiques de cet exil des musulmans d'Espagne à Tlemcen (Algérie)?

Les hypothèses que nous proposons sont le transfert de la mémoire collective esthétique et artistique, de la mémoire socio historique et de la mémoire sociolinguistique.

\section{RAPPEL HISTORIQUE}

Ces musulmans, venus des grands centres urbains d'Espagne, ont préféré l'exil vers les pays du Maghreb. Leurs apports socio culturels ont contribué à l'amélioration des conditions sociales de la population de la ville de Tlemcen et à l'attribution du titre de « La perle du Moghreb ». Les savants et les mystiques, en particulier Sidi Abou Médiène, ont contribué favorablement à l'amélioration de l'image de la localité, devenue par le temps, un centre de rayonnement culturel et un berceau du savoir et de la culture arabo- musulmane. La mémoire historique rappelle l'accueil des Andalous et surtout Boabdil ${ }^{4}$, un des derniers rois d'Espagne musulmane, décédé et enterré à Tlemcen.

La population musulmane d'Espagne était, majoritairement, constituée d'artistes et d'artisans, voire des maîtres de divers métiers. Ils sont arrivés avec leur art et leur savoir -vivre. Parmi ces derniers, il y avait des maçons dont la famille Samarchiq ${ }^{5}$, ceux qui apportèrent, malgré eux, les secrets de leur métier et leur art, et qui ont fait de la ville d'accueil un grand musée.

\section{L'EXIL POSITIF : NOTION ET EXPRESSION}

Le départ des centres urbains de l'al-Andalus et l'exil vers les villes maghrébines ont fait de cette population musulmane, chassée d'Espagne, une catégorie socio professionnelle d'exception. Des familles en-

4 Mûhammed XII de Grenade, de son vrai nom Abû Abdellah, surnommé Ezzoghbi (infortuné) : né en 1459 à Grenade et meurt en 1533-34 à Fez. Certains disent décédé à Tlemcen et entérré dans le cimetière des zianides dont la tombe a été découverte au début de la colonisation de la ville de Tlemcen (entrée en 1836), alors la pierre tombale aurait disparue.

5 Nous avons enregistré plusieurs réalisations, œuvres de la famille de Samarshiq. 
tières sont arrivées dans un pays musulman pour vivre dans une ambiance socioreligieuse de convenance et non de compromission. Immédiatement arrivés, ces chefs de famille ont recomposé la population locale avec les autochtones, avant l'arrivée des kouloughlis, ces anciens ottomans, de la corporation des janissaires, mariés à des femmes algériennes. Depuis, ces exilés se sont confondus avec les « hadars » (civilisés) dont la majorité est d'origine andalouse.

Le mot « exilé » est utilisé dans un sens plus large que l'on pense, puisqu'il désigne des situations, à plusieurs niveaux :

- La situation d'abandon du pays natal.

- La situation de la recherche d'un pays d'accueil.

- La situation de la recherche d'un refuge pour éviter toute forme de persécution religieuse.

- La situation de la pression psycho-sociale et politique.

- La situation du choix de l'auto-exil.

- La situation de l'abandon du pays de l'expérience professionnelle.

Nous ne voulons point insister sur les conditions et les situations qui ont poussé ces musulmans au départ forcé du pays où ils ont abandonné leurs souvenirs, leur mémoire et leur passé et celui de Tlemcen. Les souvenirs des exilés sont uniquement des souvenirs qui rappellent un retour vers l'enfance. Ces souvenirs constituent, principalement, les mémoires sociale et culturelle qui restent, pour nous, une matière à explorer, voire exploiter. Ce travail nous conduit à effectuer des recueils de témoignages, qu'ils soient dans des ouvrages généraux ou des ouvrages autobiographiques.

Le vécu, à travers la mémoire et l'histoire, reste spécifique et exceptionnel à ces exilés. Nombreuses sont les familles qui sont restées sur la péninsule ; mais ils ont opté pour un autre choix : le christianisme, voire un changement de noms patronymiques. Pour cette catégorie, l'analyse du processus de leur conversion demeure un sujet tabou ; mais pour les sociologues et les anthropologues, il serait d'une grande importance pour une éventuelle étude socio anthropologique, se rapportant à une population qui remonte aux $\mathrm{XV}^{\grave{e}}$ et $\mathrm{XVI} \mathrm{I}^{\mathrm{e}}$ siècles. 
Aujourd'hui, nous vivons, à travers les études déjà entamées, des moments de cumulation dans le discours de l'exception et de prise de conscience cumulative de cette situation. A notre sens, cet exil serait d'un grand apport pour les princes de l'ancienne capitale du Maghreb central, Tlemcen, devenue, depuis, un centre de rayonnement culturel et un foyer du savoir et de la culture arabo- musulmane, voire une civilisation arabo mauresque.

Loppression religieuse fut exprimée par la chasse des musulmans. Ces derniers se sont mis au service de l'autorité du pays d'accueil, mais en contre-partie, il n'y a pas eu de quête d'identité puisque l'intégration sociale et culturelle a été, pour eux, dans de bonnes conditions. Les Andalous sont arrivés avec leur culture et leur savoir-faire que les princes du royaume Abdelouadide, puis Zianide, ont adopté dans leur quotidien et celui de leur population dans un but précis : l'amélioration des conditions de vie sociale. Celles-ci ont touché le monde du travail (avec l'artisanat et les différents métiers) et l'habitat ( ce que nous appelons aujourd'hui la maison médinale, avec un cachet traditionnel). A titre d'exemple, nous évoquons le nom des Samarcheq, une famille de maître - maçons ${ }^{6}$.

L'exil n'a pas touché, uniquement, le corps des métiers puisque plusieurs lettrés et savants andalous dans les différentes disciplines sont arrivés à Tlemcen où une descendance se vante, à ce jour, de leur origine et de leurs ancêtres, de valeureux personnages, devenus, par le temps, des notables locaux.

A leur arrivée, les Andalous n'ont jamais senti l'exil ni le problème d'accueil ou d'intégration sociale et culturelle. Aucune perte de l'identité n’a été enregistrée, ni de crise identitaire, ni de décadence, ni de régression et ni le besoin d'exister dans le pays d'accueil; mais au contraire, ils ont enregistré la solidarité sociale et culturelle, l'intégration facile de la grande population locale, au sein d'une communauté musulmane, des améliorations du quotidien qui touchent, principalement :

- Le port du haik (un drap blanc, utilisé par les femmes) ${ }^{7}$.

6 Une conférence sera donnée au CRASC-Oran le 27 mai 2010 : « La famille Tlemcenienne (SARAMCHEQ) et ses réactions architecturales. »

7 Entretien avec F. Benahmed et F. Z. M'hidi 
- La distribution et l'esthétique de la maison ${ }^{8}$.

- La distraction et la détente de la population locale9.

- La production poétique et artistique : nous parlons du hawfi, du hawzi, du 'arûbi et du gharbi ${ }^{10}$.

- La représentation de la vie sociale et culturelle des princes ${ }^{11}$.

\section{LE TRANSFERT DE LA MEMOIRE}

La société est constituée de groupes sociaux. Leurs composantes sont homogènes et présentent des aspects sociaux et collectifs. L'approche sociologique de la mémoire nous renvoie à l'œuvre de Maurice Halbwachs. A titre indicatif, l'utilisation du concept de «la mémoire collective » remonte à 1939 (il serait utilisé dans un article intitulé, « la mémoire collective chez les musiciens»).

Il s'agit de la mémoire collective, en plus de l'esthétique et de l'artistique $^{12}$. Cet exil présente, en effet, des caractéristiques distinctes, incitées par le climat communautaire (le religieux et le coutumier), constituant les marqueurs suivants :

- L'habitat ${ }^{13}$.

- Les habits ${ }^{14}$.

- La gastronomie ${ }^{15}$.

- La distraction et la musique ${ }^{16}$.

- La solidarité mutuelle ${ }^{17}$.

- L'intégration sociale et culturelle.

- Le travail domestique ${ }^{18}$.

8 Entretien avec Hadj Mustapha Bendimerad, coiffeur et musicien.

9 Entretien avec Salah Boukli Hassan, maitre- artisan et artiste- maitre musicien, président de l'association Qortobia (Tlemcen).

10 Entretien avec Mohammed Souhil Dib.

11 Entretien avec Si AbdelqaderMahdad.

12 Cf. Hadri Boughrara, Voyage sentimental en musique arabo-andalouse, Paris, Méditerranée-Edif, 2002.

13 Entretien avec Hadj Abdellah Boutaleb.

14 Entretien avec Hadja K. Bounguedih Bent Hadj Ahmed.

15 Entrtetien avec F. Z. M'hidi et F. Benahmed.

16 Entretien avec Salah Boukli Hassan.

17 Entretien avec Hadj Mohamed Bakhti.

18 Entretien avec Hadj Abdellah Boutaleb. 
- L'artisanat.

- Les rituels religieux et mystiques ${ }^{19}$.

- Les rituels festifs.

L'histoire et la mémoire se distinguent. Leurs caractéristiques distinctes demeurent en relation avec le temps et l'espace ; elles se complètent. Peut-être, l'une dépend de l'autre. Dans notre approche, la mémoire et l'histoire sont nécessaires et que la première est indispensable à la détermination de l'identité. Cette identité est collective puisqu'elle se rapporte au vivre ou savoir vivre ensemble. Dans le second cas, il s'agit d'une harmonie dans la vie sociale et culturelle.

Si les souvenirs sont collectifs pour un groupe social, la mémoire est donc collective. On ne peut pas parler de mémoire sans souvenirs. Alors, les souvenirs ne peuvent pas exister sans rappeler la mémoire qui se formule par une perception d'un fait ou d'un événement dans un temps révolu. Les recherches nous permirent de parler de mémoire individuelle qui ne serait qu'une part d'un ensemble de souvenirs, collectivement, constitués. Nombreux sont les auteurs qui insistent sur les souvenirs collectifs pour reprendre leur mémoire individuelle, qui n'est qu'une part de la mémoire collective, marquée, principalement, par le vécu du groupe social.

\section{LES MEMOIRES}

Dans le cadre de l'histoire et de la mémoire, nous rappelons toutes les tentatives de se souvenir, de parler et d'insister sur ces us et coutumes adoptées par les Andalous que la population tlemcenienne a pu acquérir par la transmission de génération en génération. Aujourd'hui, nous désirons expliquer et faire comprendre ce que furent les années de l'âge d'or de cet héritage d'al-Andalus ${ }^{20}$.

Nous nous sommes retrouvés devant une situation qui nous impose cette volonté qui vise, principalement, la prise de conscience, en étroite relation avec nos recherches entamées depuis quelques décennies et qui

19 Entretien avec Hadj Mohamed Bakhti et Hadj Abdellah Boutaleb.

20 Cf. E1 Hassar Ben Ali, Tlemcen. Cité des grands maîtres de la musique arabo-andalouse, Alger, Editions Dalimen, 2002. 
se rapportent à l'histoire et à la mémoire de ces Andalous, intégrés dans la société tlemcenienne, avec beaucoup de facilités. Tous les chercheurs des deux rives se sont penchés vers un même point d'attraction : l'histoire et la sociologie andalouses. Ces deux points font la mémoire de ce peuple exilé au Maghreb. Chacun peut raconter une partie de cette histoire qui n'est que la mémoire sociohistorique dont le recoupement est nécessaire. Les souvenirs se recoupent, comme pour la mémoire collective ; mais ils se complètent par la diversité des expériences de chacun de ces exilés.

Dans la maison traditionnelle, plusieurs indices démontrent que des éléments architecturaux nous renvoient aux détails relatifs à la distribution des espaces et à l'esthétique, d'inspiration andalouse. Ces mêmes éléments fournissent des repères d'un art qui ne s'explique que par l'histoire et la mémoire. Ils auraient, sans nul doute, participé à l'amélioration de la vie socioculturelle et contribué aux changements, enregistrés dans les différents domaines du vécu. Alors, ce changement avait, également, donné un sens au savoir vivre qui touche, directement, la vie citadine et surtout celle de l'intérieur afin d'assurer la vie intérieure aux femmes et une partie de la vie du dehors aux hommes. "Wasteddar » (la cour intérieure), à titre d'exemple, est un centre de vie sociale et culturelle des femmes. L'élément déterminant est cette vie de l'intérieur où se dégagent les principes élémentaires de l'énergie, du respect mutuel, de la considération et la convivialité d'une population de maison et non à maison. Les femmes, comme les hommes, s'attachent beaucoup aux principes du respect de l'intérieur que les gens associent à la notion du « harem », un vocable d'origine est arabe.

Dans la maison traditionnelle et médinale, nombreux sont lesTlemceniens qui organisaient des soirées musicales, dans le cadre des rituels festifs familiaux. Il s'agit de la « walima », la célébration des événements familiaux dont le mariage (el 'eurss), la naissance d'un nouveau - né (essaba') et la circoncision (t-hara). Plusieurs mots arabes proviennent de l'Andalousie qui nous interpellent, comme Lachachi Hadj Omar, pour parler des apports en linguistique, voire sociolinguistique.

Ces soirées festives et conviviales sont animées par des maîtres musiciens, communément appelés « shûyûkh » (pluriel de sheikh). Malgré l'animation des maîtres, l'apprentissage du répertoire musical est de ri- 
gueur puisqu'il vise, essentiellement, la transmission du savoir musical de la « nûba », réputée pour son caractère modal. Un corpus est constitué pour une éventuelle publication dans le domaine de la sociolinguistique et de la dialectologie.

Il faut noter que l'apprentissage du répertoire tlemcenien se fait, impérativement, par la méthode orale. D'ailleurs, tout est exigeant : l'apprentissage par la transmission orale (l'oralité est de rigueur) demande, veut et exige une douzaine d'années et plus pour une solide formation, et surtout sans discontinuité. Cette formation continue se complète par le contact direct et permanent avec, au moins, un sheikh qui, en maître spirituel, lui inculque la tradition et ses modes rituels de chaque «nûba $»^{21}$.

Chaque élève - musicien doit, impérativement, connaître un certains nombre de choses élémentaires de la musique andalouse dont le vocabulaire musical et la structure de chaque «nûba »22. Pour rappel, la musique andalouse est une musique savante et ses principes sont d'ordre conventionnel. Le nombre de "nûba » est fonction du nombre d'heures de la journée et de la nuit ${ }^{23}$. Sans maître - musicien, aucun élève ou disciple ne pourra accéder aux finesses et aux raffinements musicaux qui sont, d'ailleurs, de style et de genre. Cette tradition est d'origine andalouse, comme le veut l'histoire de cette musique dite traditionnelle et ancestrale ${ }^{24}$.

L'histoire et la mémoire nous assistent pour parler de la diffusion de la musique andalouse, et surtout de l'école de Tlemcen, objet de notre recherche. L'interprétation musicale du texte pourra être considérée comme une forme d'un retour rituel de la tradition orale, cette oralité qui remonte à plusieurs générations. Ce phénomène musical d'amplification, de diffusion et de transmission de la mémoire musicale esthétique et artistique est la source d'un aspect technique qui n'est point nouveau pour les musiciens et les profanes. Il s'agit des variantes — textes et musique- pour parler de la diversité des versions.

21 Cf. Mahmoud Guettat, La musique classique du Maghreb, Paris, Sindbad, 1980.

$22 C f$. Koceil Amazigh, «Deux grands maîtres de la musique classique dans la tourmente de la nuit coloniale: Cheikh Mohammed Dib, Cheikh Ghouti Dib », Tlemcen, Association du Vieux Tlemcen, 1985.

$23 C f$. Annexe n. ${ }^{\circ} 1$.

$24 C f$. Annexe n. ${ }^{\circ}$ 2. On retrouve les musiciens d'origine arabo-musulmans et ceux d'origine juive. 
A cet effet, nous évoquons le hawfi,le hawzi, le 'arûbi' ${ }^{25}$ et le gherbi et nous notons la valeur esthétique des différents enregistrements dont les critères d'évaluation sont :

- La qualité musicale.

- Le respect de l'échelle musicale.

- Le respect de la ritualité dans la «nûba ».

- Le degré de maîtrise des instruments de musique.

- La constitution de l'orchestre.

- La place de l'instrument de musique du maître par rapport à la composante de son orchestre.

Par l'apprentissage, il faut entendre la transmission des traditions orales que le maître - musicien corrige, au fur et à mesure, par des petites retouches, rituellement et musicalement écoutables. C'est un encouragement de l'élève - musicien ou le disciple. Cet encouragement se confirme, par l'histoire et la mémoire et par la chaîne de transmission, effective grâce à une liste de maîtres - musiciens qui, alors à notre disposition, remonte à la fin de l'époque ottomane.

Cette transmission orale se présente comme une interaction et une dialectique ${ }^{26}$ que l'élève doit acquérir auprès de son maître - musicien. Cette pratique, devenue primordiale, rejoint les pratiques socioculturelles, répandues au sein de la société tlemcenienne. Elle demeure similaire aux pratiques des maîtres - artisans dans les différents métiers de l'artisanat traditionnel de l'aire géoculturelle que nous étudions. Nombreux sont les personnes qui l'associent à un legs andalou.

Il s'agit d'une vitalité conceptuelle et d'un dynamisme socioculturelle dans tous les domaines de la vie sociale et culturelle locale et non une rivalité dans l'exercice, dans la profession et dans le métier.

25 Entretien avec Mohamed Souhil Dib.

26 Dans le sens de bien mener, voire bien conduire et diriger le dialogue ou la discussion entre le maître et son élève ; et ceci, en vue de mener un raisonnement, très rigoureux, destiné, musicalement parlant, à emporter l'adhésion des interlocuteurs. 


\section{Annexes}

\section{Annexe n. ${ }^{\circ} 1$}

Les noms des heures de la journée et de la nuit
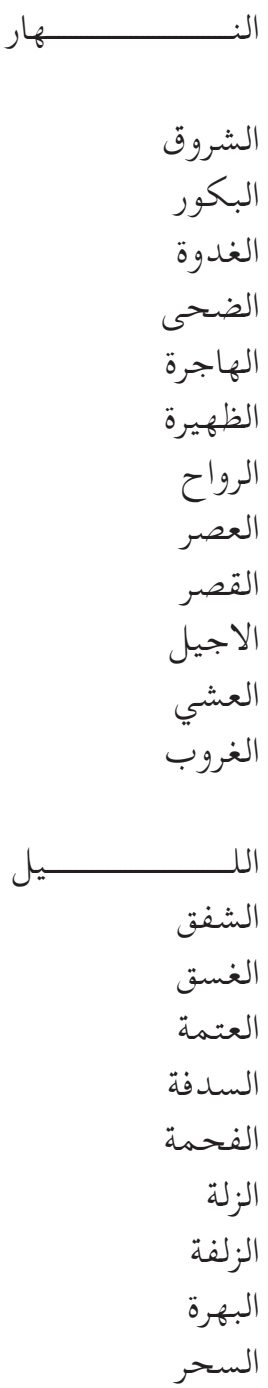


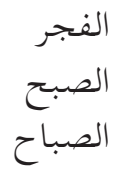

Annexe n. ${ }^{\circ} 2$

Liste des musiciens de l'école de Tlemcen
Aboura Si Mohamed (1890-1958)
Attar Abdelkader (1872-)
Baghdadli Hadj Hamadi (I797- I867)
Bedjaoui Mohamed (1904- 36)
Bekhchi Abdelkader (I86o- I918)
Bekhchi Benaouda (1880-1924)
Bekhchi Hassene (1882-1935)
Bekhchi Omar (1884-1958)
Belaghdji Abdeni (I883-1922)
Belattar Si Mostefa (1886-)
Belattar Sid' Ahmed (1897-)
Belhachemi Hamou (I86I-I9oI)
Belkhodja Mustapha (1917- 68)
Bellahcene Mohammed (1885-1950)
Benaboura Si Mostefa (1875-1965)
Benaboura Sid' Ahmed (188I-1965)
Benchenhou Ghouti (1880- 1934)
Benchenhou Sid' Ahmed (1873- I923)
Benattou Ménouar (I847-1899)
Benchaabane Mohammed (1853-19I4)
Bendi Moussa Mohammed (1815-70)
Benguerfi Mohamed (1916-)
Benkelfat Sid' Ahmed (1875-1930)
Benkebil Boumédiène (1899-1976)
Benkhobza Braham (I829-)
Benmansour Abdellah (1900-I955) 
Ben M'saib Mohammed

BenmansourZine El Abidine (I9I2-)

Bentabet Mohammed (I897-I947)

Bentata Mohammed (1839-1907)

BentchoukKaddour (1890-1942)

Bentriki Ahmed

Bensahla Boumédiène

Bensahla Mohammed

Bensaid Ghomari (I855-)

Bensaid Issâac (I84I-I9II)

Bensmail Mohammed (1884-I947)

Benyadi Si Mohammed (I8866-I952)

Benyacob Braham (I827-)

Berrahma

Bessaoud Hamed (I878-I909)

Bessaoui Mohamed Séghir (1876-1934)

Bouali Mohamed (I9I7- 98)

Bouali Si Ghouti (I874-I934)

Bouchama Sid' Ahmed (1897-1977)

Boukharouba Mohammed El Kébir (I8I9-92)

Boukli Hassene Hadj Hamadi (I809-59)

Charif HadjHammou (I8I5-84)

Chyerif Benmoussa Mohamed (I877-1947)

Chicha Mohamed (1870- 1925)

Chikhi Mohammed (1892-I942)

Chouikhi Khaled

Choukchou Braham Mohammed (I84I-I902)

Dali Ali Abdelkrim (I9I4-78)

Dali Yahia Mohammed (I894- I946)

Dib Abdelkader (I829-7I)

Dib Ghouti (1863-I919)

Dib Mohammed (I86I-I9I5)

DibMustapha (I857-78)

Djian Nassim (1847-84)

El Bar Makhlouf (1848-I9I3) 
E1 Draï Braham (1879-I964)

El Draï Moïseould Braham (-I96o)

En Nadjar Braham (I829-)

Fidah Moro Djelloul (I850-90)

Ghali Benkemoun (1846-96)

Ghomari Mohammed (1842-)

Hadj Amara Mohammed (I8o8-68)

KaidSlimane Mohammed (I892-I955)

Kelaidji Aberahim (1880-68)

Kelaidji Si Mohammed (I90I-)

Kazi Ghouti (-r963)

Kermouni Serradj Abdelkader (I866-I946)

Lazzouni Ahmed

Lazzouni Ahmed ould Lazzouni (1885-1953)

Lazzouni Ahmed ould Mohammed (I879-)

Lazzouni Ali (1805-)

Lazzouni Benali (I854-I9II)

Lazzouni Kada

Lazzouni Mohammed (I8I9-95)

Lazzouni Mohammedould Mohammed (1853-1916)

Lazzouni Mohammed ould Ali (I872-)

Lazzouni Mokhtar

Liahou Ben youcef ( $18 \mathrm{II}-56)$

Liahou eL Ankri (I8I4-)

LiahouLadri (I82I-54)

Malti Abdelghani (I92I- I99I)

Mansour Tani Mohammed (I845- I9I2)

Medeghri Moulay Ahmed (I843-I925)

Medelci Ghouti (I847-I92I)

Médioni Ichoua (1829-92)

Médioni Israél (1858-79)

Médioni Mouchi (I842-)

Médjadi Mohammed (1899-)

Mesmoudi Si Daoudi (I849-I9I3)

Mir Hassaine Ali (I8I3-93) 
Mouchi Chloumou (1853-98)

Moula Djilali (I893-I947)

Rouche Mouchoi (I865-I922)

Rouche Makhlouf (1858-I931)

Saber Zenagui Abdelkader (I899-)

Saber Zenagui Abdelkader ould Benali (I876-ı926)

Said Mohammed (I849-I903)

Saoud El Médiouni (I8I4-)

Sari Abdeslam (I876-I959)

Sari Abdeslam (I890-I964)

Sari Ahmed dit Redouan (I9I4-2002)

Sari HadjLarbi (1872-I964)

Sari Mohammed (I9II-)

Sari M'hammed (I9Io-82)

Sarmachik Boumédième (I908-)

Sayagh Isaac (I843-I9o6)

SekkalAbdelkader (I863-I9I5)

Sekkal Mohamed dit Abderahmane (I9Io-85)

Settouti Abdelhamid (1902-40)

Senouci Bereksimustapha (I9I9-)

Tabet Tétma (I89I-I962)

Teboul Simah (1828-)

Terki Hassaine Mohammed Seghir (1905-78)

Tchouar Si Mohamed (I865-I942)

Touati Chaloum (I829-98)

Touati Isaac (I847-I9I2)

Zenagui Mohammed (1829-59)

Zerrouki Bachir ould Djilali (1924-)

Zerouki Djilali (I839-)

Ziani Chérif Djilali (I873-I939)

Zianimoulay Djilali (I85I-I933) 
